\title{
Arm retraction dynamics of entangled star polymers: a forward-flux sampling method study
}

Article

Accepted Version

Zhu, J., Likhtman, A. E. and Wang, Z. (2017) Arm retraction dynamics of entangled star polymers: a forward-flux sampling method study. The Journal of Chemical Physics, 147 (4). 044907. ISSN 0021-9606 doi:

https://doi.org/10.1063/1.4995422 Available at https://centaur.reading.ac.uk/71476/

It is advisable to refer to the publisher's version if you intend to cite from the work. See Guidance on citing.

To link to this article DOI: http://dx.doi.org/10.1063/1.4995422

Publisher: American Institute of Physics

All outputs in CentAUR are protected by Intellectual Property Rights law, including copyright law. Copyright and IPR is retained by the creators or other copyright holders. Terms and conditions for use of this material are defined in the End User Agreement.

www.reading.ac.uk/centaur 
Central Archive at the University of Reading

Reading's research outputs online 
Arm Retraction Dynamics of Entangled Star Polymers: A Forward-Flux Sampling Method Study

\author{
Jian Zhu, Alexei E. Likhtman and Zuowei Wang*1 \\ Department of Mathematics and Statistics, University of Reading, \\ Reading RG6 6AX, UK
}

The study of dynamics and rheology of well-entangled branched polymers remains a challenge for computer simulations due to the exponentially growing terminal relaxation times of these polymers with increasing molecular weights. We present an efficient simulation algorithm for studying the arm retraction dynamics of entangled star polymers by combining the coarse-grained slip-spring (SS) model with the forwardflux sampling (FFS) method. This algorithm is first applied to simulate symmetric star polymers in the absence of constraint release (CR). The reaction coordinate for the FFS method is determined by finding good agreement of the simulation results on the terminal relaxation times of mildly entangled stars with those obtained from direct shooting SS model simulations with the relative difference between them less than $5 \%$. The FFS simulations are then carried out for strongly entangled stars with arm lengths up to 16 entanglements that are far beyond the accessibility of brute force simulations in the non-CR condition. Apart from the terminal relaxation times, the same method can also be applied to generate the relaxation spectra of all entanglements along the arms which are desired for the development of quantitative theories of entangled branched polymers. Furthermore, we propose a numerical route to construct the experimentally measurable relaxation correlation functions by effectively linking the data stored at each interface during the FFS runs. The obtained star arm end-to-end vector relaxation functions $\Phi(t)$ and the stress relaxation function $G(t)$ are found to be in reasonably good agreement with standard SS simulation results in the terminal regime. Finally, we demonstrate that this simulation method can be conveniently extended to study arm-retraction problem in entangled star polymer melts with CR by modifying the definition of the reaction coordinate. 
I. INTRODUCTION

Development of quantitative theories for predicting the dynamic and rheological properties of entangled branched polymers is of both fundamental and practical importance. In the past decades, theoretical efforts have been primarily based on the concept of tube model originally proposed by de Gennes, Doi and Edwards. ${ }^{1-3}$ Different from entangled linear polymers where reptation, contour length fluctuations (CLF) and constraint release (CR) are the main relaxation mechanisms, reptation in branched polymers is strongly suppressed due to the effectively localized branch points. In the simplest case of symmetric star polymers, the stress relaxation is conjectured to proceed via CLF or arm retraction by which the free end of an arm retracts inward along the primitive path to escape from the original tube segments and pokes out again to explore new tube. Since arm retraction is entropically unfavorable and so thermally activated, this process can be formulated as a first-passage (FP) problem or Kramers problem. ${ }^{4-6}$

A star arm retracting in a fixed network experiences a potential barrier theoretically described by a quadratic function $U(s)=\nu k_{\mathrm{B}} T Z s^{2}$ where $k_{\mathrm{B}}$ is the Boltzmann constant, $Z=M / M_{\mathrm{e}}$ is the number of entanglements per arm, $M$ is the arm molecular weight, $M_{\mathrm{e}}$ is the entanglement molecular weight and $\nu$ is treated as a constant. ${ }^{7}$ The fractional coordinate $s$ measures the retraction depth of the arm free end. Pearson and Helfand predicted an exponential dependence of the arm terminal relaxation time, $\tau_{\mathrm{d}}$, and correspondingly the viscosity, $\eta_{0}$, on the arm molecular weight, $\eta_{0} \sim \tau_{\mathrm{d}} \sim \exp \left(\nu M / M_{\mathrm{e}}\right){ }^{8}$ This prediction, however, shows a large discrepancy from experimental data obtained in star polymer melts due to the neglect of $\mathrm{CR}$ effects. Ball and McLeish ${ }^{9}$ took into account the $\mathrm{CR}$ effects by applying the dynamic tube dilution (DTD) hypothesis ${ }^{10}$ where the relaxed arm segments are considered to work as an effective solvent for the unrelaxed materials. Milner and McLeish further improved this theory by including the contributions of fast Rouse fluctuations at early times and solving the first-passage problem of a diffusing end monomer to retract a fractional distance $s$ to get the arm relaxation spectrum $\tau(s)$ at late times. ${ }^{4,5}$ The MilnerMcLeish theory predicts the stress relaxation of symmetric star polymer melts reasonably well, but not the dielectric or arm end-to-end vector relaxation function. It also encounters difficulty in using a single set of model parameters to describe the rheological behaviors of asymmetric star polymers with different short arm lengths. ${ }^{11}$ In recent years computational 
models based on the framework of Milner-McLeish theory have been developed for describing the linear viscoelasticity of branched polymers with arbitrary architectures and their general mixtures. ${ }^{12-16}$ These models have been shown to provide predictions in reasonably good agreement with experimental data for a variety of systems, but are facing problems in describing the linear rheology of some simple mixtures, such as the star-linear blends, especially at low fractions of star polymers. ${ }^{16,17}$ Therefore more quantitative theories that can simultaneously predict different dynamic and rheological properties of entangled branched polymers are still highly desired. The development of such theories requires the analytical solution of the multi-dimensional FP problem of arm retraction. ${ }^{18}$

On the other hand, the coarse-grained slip-link or slip-spring (SS) simulation models have demonstrated strong potential in describing dynamics and rheology of entangled polymers. ${ }^{19-28}$ For example, the single-chain slip-spring model developed by Likhtman ${ }^{25}$ can provide simulation results on multiple experimentally measurable observables, such as neutron spin echo, linear rheology, dielectric relaxation and diffusion. Using a limited number of fitting parameters, the predictions of this model match the results obtained from both experiments and molecular dynamics (MD) simulations on linear and symmetric star polymers very well. ${ }^{26,29-31}$ The SS model serves as an intermediate between tube theory and MD simulations. As a discrete model, it not only naturally builds in all the relaxation mechanisms of the tube model, but also carries more system details, such as explicit polymer chains and entanglements $^{32}$. At a higher level of coarse-graining, the SS model is significantly more efficient than MD simulations using bead-spring polymer model, which is of great advantage in the study of branched polymers. Furthermore, the slip-spring model can separate the contributions from different relaxation mechanisms by enabling some of them while disabling others. This is particularly helpful for examining assumptions made in current theoretical models and providing valuable information for developing more quantitative models. One typical application is to evaluate the magnitude of constraint release effects by comparing simulation results obtained from entangled polymer systems with and without CR.

Since deep arm retractions are rare events due to the high entropic barrier, the time and length scales accessible to standard slip-spring simulations are still much shorter than those in well-entangled experimental systems where the tube models are supposed to work best. Similar problems have also been seen in brute force simulations of many other rare events, such as crystal nucleation ${ }^{33,34}$, biological switches ${ }^{35}$ and protein folding ${ }^{36}$. The required 
computational time may take up to several decades. ${ }^{37}$ Advanced numerical techniques, such as the umbrella sampling ${ }^{38}$ and transition path sampling ${ }^{39}$ methods, have to be employed to accelerate the simulations. Recently the forwards flux sampling (FFS) method has been proposed $^{35,40,41}$ and proven to be successful in molecular dynamics and Monte Carlo (MC) studies of rare events. ${ }^{37,42}$

In this work, we will combine the FFS method with the slip-spring model for studying the dynamics of entangled symmetric star polymers. This is a proof-of-concept work. To our knowledge the only reported work on applying the transition path sampling methods to study entanglement dynamics is the FFS simulation of Rouse chains in the regime relevant to arm retraction dynamics. ${ }^{18}$ We will mainly focus on the systems without constraint release for the following reasons: 1) It is relatively convenient to implement the FFS method and find an appropriate reaction coordinate in the non-CR systems; 2) The terminal relaxation times in the systems without $\mathrm{CR}$ are much longer than those with $\mathrm{CR}$, allowing us to test the computational efficiency and limit of the combined method; 3) Reliable simulation data on the FP times of arm retractions without $\mathrm{CR}$ are highly desired for examining analytical solutions of the multiple-dimensional Kramers problem ${ }^{18}$; 4) The extension of the method developed in the non-CR case to the CR case is fairly straightforward, as will be shown in Section V. With an optimized selection of the reaction coordinate, which is the index of the monomer that the innermost slip-link sits on, we first validate the proposed simulation method by producing simulation results on the terminal relaxation times $\tau_{\mathrm{d}}$ of mildly entangled star arms up to 8 entanglements in good agreement with those obtained from SS model simulations. The FFS simulations are then extended to longer arms with lengths up to 16 entanglements and so reach $\tau_{\mathrm{d}}$ values about 6 decades beyond that accessible by brute force simulations (from $6 \times 10^{6}$ to $3 \times 10^{12} \mathrm{SS}$ unit time). The FP times of other original slip-links along the arm can be calculated using similar FFS simulations as for the innermost one, which consequently provides the entire arm relaxation spectrum $\tau(s)$. Moreover, we propose a numerical route to construct the arm end-to-end vector correlation functions, $\Phi(t)$, and stress relaxation functions, $G(t)$, from the discrete data stored at each interface during the FFS runs. Such time correlation functions are still not widely addressed in the FFS studies, but some relevant discussions could be found in the literatures for the FFS ${ }^{43,44}$ and weighted ensemble methods. ${ }^{45-47}$ Our simulation results will contribute to the development of theoretical models for describing the dynamics of entangled branched 
polymers and also the general first-passage problems in multi-dimensional systems. The simulation methodology developed in this work should also be applicable to the study of rare events in other scientific areas.

The rest of this paper is organized as follows. In Section II, we introduce the single-chain slip-spring model for entangled star polymers in the absence of CR. The detailed description of the combined FFS and SS model is given in Section III. The simulation results obtained in the non-CR systems are presented and discussed in Section IV, including the terminal relaxation times $\tau_{\mathrm{d}}$, the arm retraction spectra $\tau(s)$ and the numerical route for constructing $\Phi(t)$ and $G(t)$. In Section V, the simulation method is extended to study the arm retraction dynamics of star polymers in the presence of CR. We draw conclusions in Section VI.

\section{SLIP-SPRING MODEL FOR ENTANGLED SYMMETRIC STAR POLYMERS}

\section{A. Model Description}

In the single-chain slip-spring model for entangled symmetric stars, each star arm is represented by a Rouse chain with $N+1$ monomers linked by $N$ harmonic springs ${ }^{25,48}$ as shown in Fig. 1. One end monomer with index 0 of the chain is treated as the branch point which is fixed in space, while the other end with index $N$ moves freely. The topological constraints on the arm are modelled by a set of virtual springs each of $N_{\mathrm{s}}^{\mathrm{SS}}$ beads. Each virtual spring has one end connected to the Rouse chain by a slip-link that can slide along the chain, and the other end, called anchor point, is fixed in space. The slip-spring model effectively assumes a binary picture of entanglements, which is qualitatively supported by recent MD simulation studies. ${ }^{49-51}$ There is on average one slip-spring every $N_{\mathrm{e}}^{\mathrm{SS}}$ monomers. The values of $N_{\mathrm{e}}^{\mathrm{SS}}$ and $N_{\mathrm{S}}^{\mathrm{SS}}$ are adjustable for describing the intensity of entanglements. It should be noted that $N_{\mathrm{e}}^{\mathrm{SS}}$ is not necessarily equal to the entanglement length $N_{\mathrm{e}}$ used in tube theory. Their relation will be discussed in Sec. IV B. To be consistent with previous publications, ${ }^{25,30}$ we choose $N_{\mathrm{e}}^{\mathrm{SS}}=4$ and $N_{\mathrm{s}}^{\mathrm{SS}}=0.5$. Other parameters, such as the bead friction coefficient $\zeta_{0}$, the average bond length $b$ of the Rouse chain, the temperature $k_{\mathrm{B}} T$ and consequently the time scale $\tau_{0}=\xi_{0} b^{2} / k_{\mathrm{B}} T$, are all set to unity.

The Hamiltonian of the SS model is determined by the potential energies of both the 


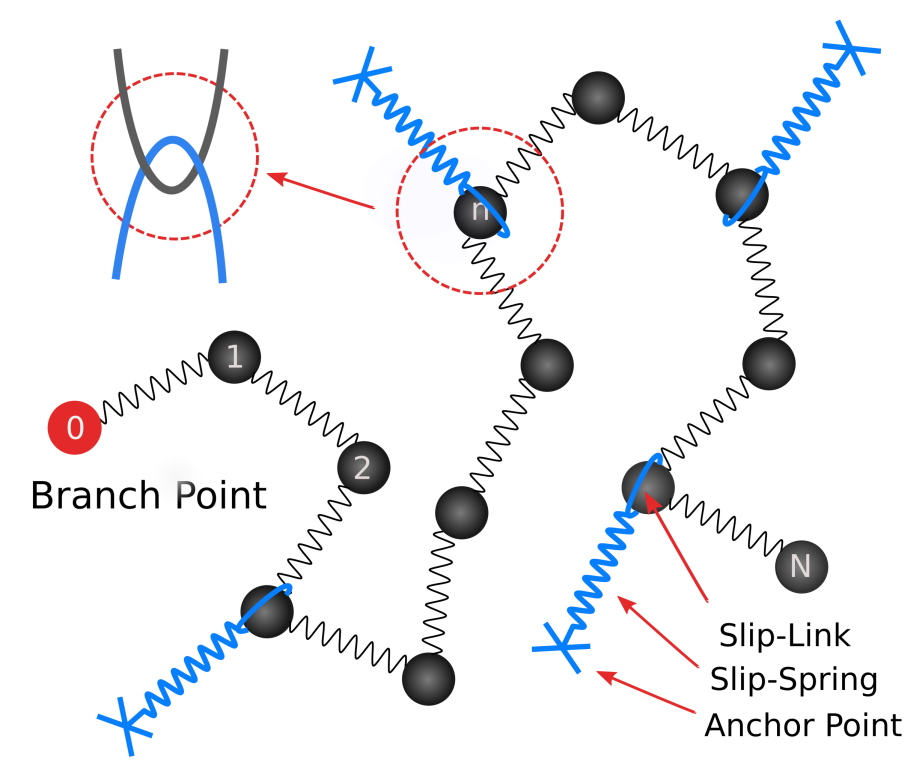

FIG. 1. Sketch of the single-chain slip-spring model for one arm of a symmetric star. The end monomer 0 represents the branch point which is fixed in space.

harmonic bonds of the Rouse chains and the virtual springs. The trajectories of the Rouse monomers are obtained by solving their Langevin equations of motion numerically using an integration time step size of $\Delta t=0.05 \tau_{0}$. In the original slip-spring model, ${ }^{25,29,30}$ the sliplinks are assumed to travel continuously along the straight lines between adjacent monomers and so can sit anywhere on the chain. In a later version of this model, ${ }^{31}$ the slip-links move discretely by hopping from one monomer to one of its nearest neighbors with the acceptance rate controlled by a Metropolis Monte Carlo (MC) algorithm. The long-time behavior of the system is not sensitive to the details of the slip-link motion. For simplicity and computational efficiency, we employ the discrete motion approach in the current work. One Monte Carlo hopping motion is attempted on average per slip-link at each time step. It has been found recently by Shivokhin et al. that the slip-springs themselves could make non-negligible contributions to the effective friction experienced by the Rouse chain when moving along the tube, because the virtual springs with finite spring constant effectively restrict the excursion volumes of the slip-links and so reduce their successful rate to hop onto adjacent monomers. ${ }^{52}$ As a consequence, an effective monomeric friction coefficient, $\xi_{\text {eff }}\left(>\xi_{0}\right)$, should be used instead of $\xi_{0}$ when mapping the simulation results of the slip- 
spring model to experimental data. But a constant change in the $\xi$ value will not affect the discussions in this work, as all the data analysis and comparison are carried out within the slip-spring model framework. The effect of slip-link friction could be reduced by increasing the number of MC hopping attempts per time step at the price of higher computational cost. The slip-links are not allowed to sit on or pass through the branch points of the star arms. In the systems without constraint release, such as star polymers in a fixed polymer network, the destruction and creation of slip-links can only take place at the free ends of the star arms. Different from the systems with $\mathrm{CR},{ }^{25}$ the slip-links are not coupled with each other. In addition, the slip-links on the same arm are not allowed to pass over each other or occupy the same monomer. This assumption introduces an effective excluded volume interaction between the slip-links, which is consistent with the low swapping rate between neighboring entanglements as revealed in a recent MD simulation of symmetric star polymer melts. ${ }^{51}$

The previous slip-spring simulations were typically carried out in an ensemble of chains and the total number of slip-links in the system is kept constant. ${ }^{25}$ In the non-CR case, when one slip-link is deleted from a chain end, another slip-link will be added to the end of a randomly selected chain in the ensemble. For convenient installation of the FFS method, we modify the SS model for the non-CR case by simulating each entangled arm individually. The destruction of slip-links on a given arm is still incurred by the retraction of the arm free end (monomer index $N$ ), but the addition of new slip-links to the same arm end is now determined by a probability $P_{\text {add }}$ which satisfies the detailed balance condition

$$
\left(1-\rho_{\mathrm{sl}}\right)\left(P_{\mathrm{add}}+\rho_{\mathrm{sl}} P_{N-1, N}\right)=\rho_{\mathrm{sl}}\left(P_{\mathrm{loss}}+\left(1-\rho_{\mathrm{sl}}\right) P_{N, N-1}\right),
$$

where $\rho_{\mathrm{sl}}=1 / N_{\mathrm{e}}^{\mathrm{SS}}$ is the average number of slip-links sitting on each monomer. $P_{i, j}$ is the transition probability for a slip-link to move from monomer $i$ to monomer $j$ and $P_{\text {loss }}$ is the probability for a slip-spring sitting on the arm free end to be destructed after one integration time step, respectively. Eq. 1 thus represents the balance between the flux of slip-links to and from the end monomer. Assuming $P_{N-1, N}=P_{N, N-1}$ without loss of generality, Eq. 1 gives $P_{\text {add }} \approx 0.167$ for the system parameters $N_{\mathrm{e}}^{\mathrm{SS}}=4$ and $P_{\text {loss }}=0.5$. The modified SS model is validated by studying the static properties of the simulation system. 




FIG. 2. Slip-spring model simulation results (circles) and predictions of Eq. 2 (line) on the probability distribution of number of slip-links per arm, $P\left(N_{\mathrm{sl}}, N\right)$, for symmetric star polymers with arm length $N=24$.

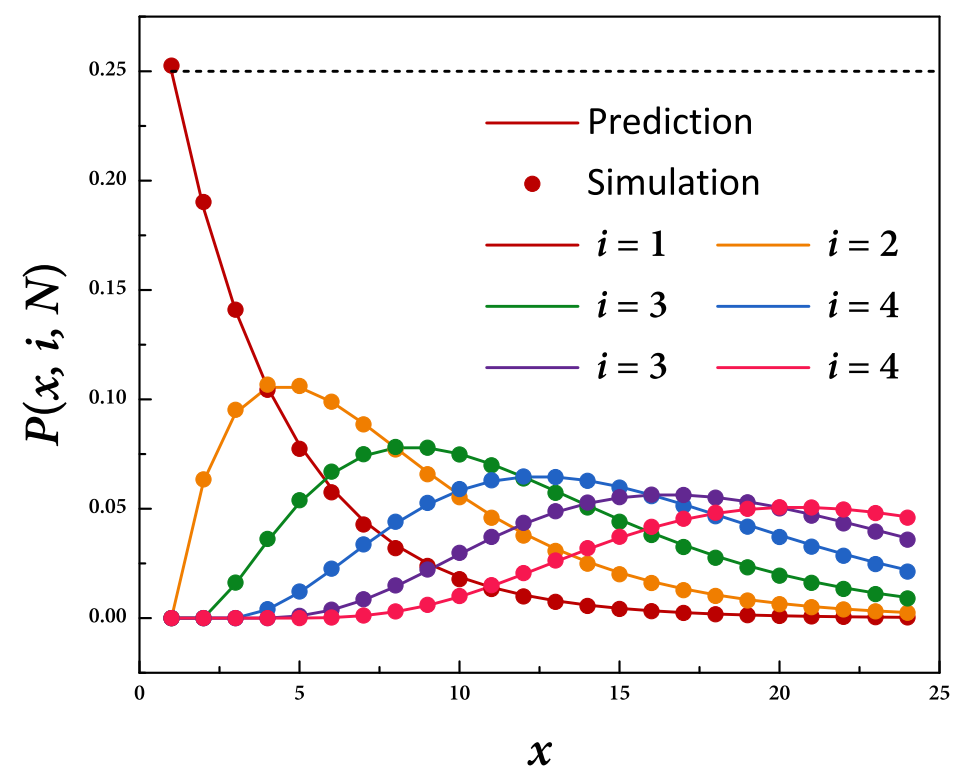

FIG. 3. Slip-spring model simulation results (symbols) and predictions of Eq. 4 (lines) on the probabilities of finding $i$-th slip-link on monomer $x, P(x, i, N)$, for the symmetric star polymers with arm length $N=24$. The horizontal dashed line shows the simulation results on the average number of slip-links found on each individual monomer. 


\section{B. Static Properties}

The static property of the slip-spring model system of entangled symmetric star polymers can be well characterized by the distribution of slip-links along the star arms. Considering the effective excluded volume interactions between the slip-links, the problem is similar to one-dimensional real gas in equilibrium. The probability distribution of finding $N_{\mathrm{sl}}$ slip-links on a star arm of $N$ monomers is simply given by

$$
P\left(N_{\mathrm{sl}}, N\right)=C_{N}^{N_{\mathrm{sl}}} \rho_{\mathrm{sl}}^{N_{\mathrm{sl}}}\left(1-\rho_{\mathrm{sl}}\right)^{N-N_{\mathrm{sl}}},
$$

where $C_{N}^{N_{\mathrm{sl}}}=\frac{N !}{N_{\mathrm{sl}} !\left(N-N_{\mathrm{sl}}\right) !}$. Fig. 2 shows the good agreement between the prediction of Eq. 2 and the SS model simulation results on $P\left(N_{\mathrm{sl}}, N\right)$ for the system with $N=24$. It can be seen that the peak value of $N_{\mathrm{sl}}$ is located at $N_{\mathrm{sl}}=6$ in consistence with the expected average number of slip-links per arm, $\left\langle N_{\mathrm{sl}}\right\rangle=\rho_{\mathrm{sl}} N=6$.

When there are $N_{\mathrm{sl}}$ slip-links on a given arm, the probability to find the $i$-th slip-link on the monomer $x$ is

$$
P\left(x, i, N_{\mathrm{sl}}, N\right)=\frac{C_{x-1}^{i-1} C_{N-x}^{N_{\mathrm{sl}}-i}}{C_{N}^{N_{\mathrm{sl}}}}, \quad\left(i \leq x \leq N-N_{\mathrm{sl}}+i\right)
$$

where the numerator is a product of the possibilities to find $i-1$ slip-links on the arm segment from monomer 1 to $x-1$ and to find $N_{\mathrm{sl}}-i$ slip-links on another segment from monomer $x+1$ to $N$. It should be noted that in the star polymer systems without CR the slip-links do not change their ordering along the star arms. In Eq. 3 the index $i$ is considered to increase from 1 for the innermost slip-link to higher values toward the arm free end. Combining Eqs. 2 and 3, we obtain the ensemble-averaged probability to find the $i$-th slip-link on the monomer $x$ :

$$
P(x, i, N)=\sum_{N_{\mathrm{sl}}=1}^{N} P\left(x, i, N_{\mathrm{sl}}, N\right) P\left(N_{\mathrm{sl}}, N\right) .
$$

Derivations of probability distributions similar to Eqs. 2 - 4 can also be found in a previous work of Schieber. ${ }^{53}$.

Fig. 3 presents the SS simulation results on $P(x, i, N)$ for the slip-links with indices $i=1$ to 6 on star arms of length $N=24$, together with the predictions of Eq. 4 . The good agreement between the two sets of data indicates that the simulation systems are in equilibrium state and the randomly assigned locations of the anchor points can well 
preserve the equilibrium distribution of the slip-links. This is also reflected by the fact that the average number of slip-links found on each individual monomer is equal to $\rho_{\mathrm{sl}}=0.25$, see the horizontal line in Fig. 3.

\section{COMBINED FFS AND SS METHOD FOR ENTANGLED STAR POLYMERS WITHOUT CR}

In the systems without $\mathrm{CR}$, the topological constraints or entanglements imposed on a target arm are released hierarchically by the retraction of the arm free end. The terminal relaxation time $\tau_{\mathrm{d}}$ of the system is defined as the average first-passage time that takes the free end of an arm to reach the branch point starting from a random initial conformation. For well-entangled star arms, $\tau_{\mathrm{d}}$ grows exponentially with the number of entanglements per arm, $Z{ }^{8}$ However, full arm retraction rarely happens at large $Z$ and so is generally not accessible by standard brute force simulations. There is also no exact analytical solution of this multi-dimensional FP problem. Therefore the forward flux sampling method introduced in Ref. ${ }^{35}$ is employed in order to study these rare events. A successful application of the FFS method on studying the FP time of 1D Rouse chain with one fixed end can be found in Ref. ${ }^{18}$.

\section{A. Forward Flux Sampling Method}

In FFS the phase space is divided by a sequence of non-crossing interfaces denoted by $\lambda_{i}$ $(i=0, \ldots, m)$, as sketched in Fig. 4(a). The starting states of the dynamic process are on the first interface $\lambda_{0}$, and the reactive or terminal states are on the last interface $\lambda_{m}$. These interfaces are defined by a reaction coordinate, which can be any parameter evolving during the process, but different choices could result in significantly different performance. More detailed discussion about the reaction coordinate is given in Sec. III B.

The FFS method is operated in two stages. In the first stage, a very long continuous simulation is performed in order to calculate the frequency $\mu_{0}$ at which the trajectory crosses the interfaces $\lambda_{0}$ and $\lambda_{1}$ in sequence. In the second stage, a set of consecutive shooting simulations are carried out from interface $\lambda_{i}$ to interface $\lambda_{i+1}$ for $i=1, \ldots, m-1$, which provide the transition probabilities $P\left(\lambda_{i+1} \mid \lambda_{i}\right)$ that a system starting from $\lambda_{i}$ will first reach 
(a)

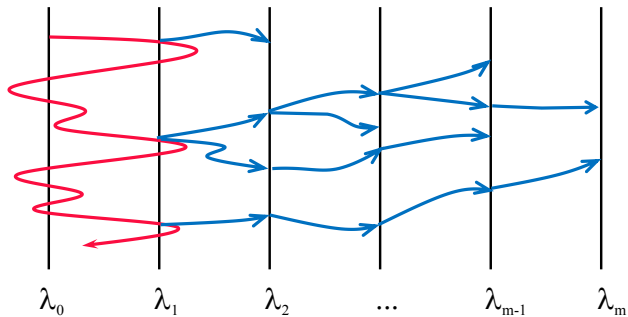

(b)



FIG. 4. (a) Schematic diagram of the FFS method. The continuous red trajectory is the continuous simulation in the first stage, and the blue trajectories are the successful shooting simulations in the second stage; (b) Algorithm for building continuous arm relaxation pathways from the piecewise shooting trajectories shown in (a).

$\lambda_{i+1}$ rather than return to $\lambda_{0}$. The first-passage time $\tau_{n}$ for the system starting from the first interface $\lambda_{0}$ and ending on the interface $\lambda_{n}(1 \ll n \leq m)$, is then given by

$$
\tau_{n}=\frac{1}{\mu_{0} \prod_{i=1}^{n-1} P\left(\lambda_{i+1} \mid \lambda_{i}\right)}, \quad 1 \ll n \leq m
$$

\section{B. Reaction Coordinate}

A key issue in applying the FFS method is the choice of the reaction coordinate. Starting from a random initial configuration, the relaxation of a star arm in the system without CR proceeds by the retraction of the arm free end along the primitive path, passing through all the original slip-links on the arm sequentially until none left between it and the branch point. The terminal relaxation time is determined by the moment at which the innermost slip-link is released. During this process, the number of surviving original slip-links, $N_{\mathrm{sl}}$, on the arm drops with time from its initial value to 0 , making it an intuitively simple choice for the reaction coordinate. Considering that the value of $N_{\mathrm{sl}}$ is statistically proportional to the length of the surviving tube or primitive path, this choice would be consistent with a recent FFS study on the FP time for the free end of a 1D Rouse chain to reach a certain distance $z$ from the fixed end where $z$ was selected as the reactive coordinate. ${ }^{18}$ The $1 \mathrm{D}$ Rouse chain 
study is closely related to the current work, because arm extension is essentially the reverse process of arm retraction. However, when using $N_{\mathrm{sl}}$ as the reaction coordinate, our FFS simulation results on the terminal arm retraction times are found to be significantly smaller than those obtained from standard SS model simulations. The problem arises from the difficulty in choosing equivalent starting states for the FFS runs. In the slip-spring model system, both the instantaneous number of slip-links and their distribution along the arm are subject to strong fluctuations, especially on the outer arm segments which undergo fast Rouse motion. In the FFS runs using $N_{\mathrm{sl}}$ as the reaction coordinate, the starting states are collected in the first-stage continuous simulation as the configurations where the number of slip-links on the arm is equal to the ensemble-averaged value of $\left\langle N_{\mathrm{sl}}\right\rangle=N \rho_{\mathrm{sl}}$. Shooting from these starting configurations, only the samples in which the values of $N_{\mathrm{sl}}$ decrease monotonically are considered to reach interface $\lambda_{1}$ successfully. This biased strategy is thus in favor of the samples where the initial slip-link densities on the outer arm segments are higher than $\rho_{\mathrm{sl}}$, because in such cases the probability to lose slip-links at short times is higher than to gain ones. Therefore a relatively large proportion of slip-links on a sample arm are released by shallow arm retractions at early times, leaving fewer than the average number of slip-links on the surviving segments of the primitive path. As a consequence, the terminal relaxation times obtained from the FFS simulations are shorter than those obtained from standard SS simulations where the ensemble-averaged initial distribution of slip-links is uniform. These results imply that the reaction coordinate should be selected close to the branch point in order to minimize the influence of the fast fluctuating arm end.

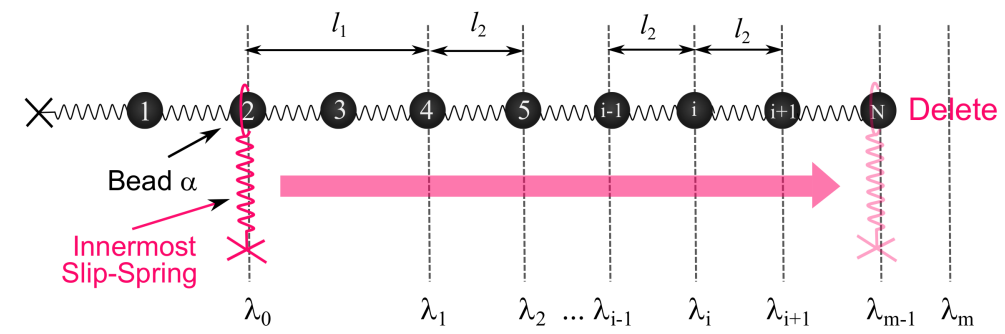

FIG. 5. Application of FFS method for studying the retraction dynamics of an entangled star arm described by the slip-spring model. The cross (Monomer 0) on the left represents the branch point that is fixed in space. The interfaces $\lambda_{i}$ (vertical lines) used in the FFS simulations are placed on the monomers of the arm.

Since the terminal arm relaxation time is determined by the release of the innermost 
slip-link from the arm free end, one can track the motion of this particular slip-link along the arm by defining the index of the monomer that it sits on as the reaction coordinate. As shown in Fig. 5 where the 3D Rouse chain is sketched as a straight line for convenience of discussion, the first interface $\lambda_{0}$ used in FFS is set on monomer $\alpha$ ( 2 in this case) where the innermost slip-link originally sits on. Any initial configuration of the confined arm in which the innermost slip-link locates on monomer $\alpha$ can be taken as the starting state of the FFS simulation. The second last interface $\lambda_{m-1}$ is placed on the outermost monomer $N$ of the arm, and the last interface $\lambda_{m}$ is right outside of the arm free end, marking the final or reactive state that the arm free end has passed through the innermost slip-link and the arm is fully relaxed. The other $m-2$ interfaces are placed on the monomers in between $\alpha$ and $N$.

According to the standard FFS method, a database containing a large number of configurations is accumulated on each interface. In the first stage of the continuous simulation, the database on $\lambda_{1}$ is a collection of configurations whose innermost slip-link lastly crossed $\lambda_{0}$ before crossing $\lambda_{1}$. In the second stage, consecutive shooting simulations are performed from interface $\lambda_{i}$ to $\lambda_{i+1}, i=1, \ldots, m-1$ using starting configurations randomly selected from the database on $\lambda_{i}$. Among the $M_{i}$ shooting samples, the ones whose innermost slip-links reach $\lambda_{i+1}$ before going back to $\lambda_{0}$ are considered as successful samples and will be stored in the database of $\lambda_{i+1}$.

\section{Simulation Details}

Apart from the reaction coordinate, the performance of the FFS algorithm can also be affected by some other factors. One factor is that the configurations saved in the database of interface $\lambda_{1}$ during the first-stage continuous simulation could be strongly correlated with each other due to the limited running time at this stage in comparison with $\tau_{\mathrm{d}}$. This may introduce systematic errors in the simulation results if the size of the database is fixed. This problem can be resolved by increasing the interval $l_{1}$ between the interfaces $\lambda_{0}$ and $\lambda_{1}$, as shown in Fig. 5, and recording configurations on $\lambda_{1}$ at a lower frequency $\omega$. For example, rather than recording every event that the innermost slip-link crosses $\lambda_{1}$ when coming from $\lambda_{0}$, one can record once for every $1 / \omega$ crossings. Another factor is the choices of the interface interval $l_{2}$ between $\lambda_{i}$ and $\lambda_{i+1}(i=1, \ldots, m-2)$ and the number of shooting 
samples $M_{i}$ from each $\lambda_{i}$ which determine the performance of the FFS in the second stage. Since $l_{2}$ controls the transition probabilities $P\left(\lambda_{i+1} \mid \lambda_{i}\right)$, a smaller $l_{2}$ is normally preferred for accelerating the shooting simulations. The number $M_{i}$ can then be chosen according to $P\left(\lambda_{i+1} \mid \lambda_{i}\right)$ and the desired accuracy.

In the current work, we take $l_{1}=2$ and $l_{2}=1$ which separate the first two interfaces $\lambda_{0}$ and $\lambda_{1}$ by one bead and then set one interface on every bead along the arm. The recording frequency $\omega$ has to be reduced for longer arms in order to reduce the conformational correlations on $\lambda_{1}$ and is empirically taken to be $\omega=1 /(N-15)$ for arm length $N \geq 16$. Since the reaction coordinate is defined by the location of the innermost original slip-link, the transition probability $P\left(\lambda_{i+1} \mid \lambda_{i}\right)$ increases with $i$ towards the arm free end. In order to achieve good statistics for the first few interfaces close to the branch point, $M_{i}$ should be large enough. A number of samples $M_{i}=40,000$ is thus used for $\lambda_{i}, i=1,2, \ldots, m-1$ in all of the FFS simulation runs. As shown in Fig. 3, there is a non-negligible fraction of initial configurations where the innermost slip-links are many monomers away from the branch point and could be released by shallow arm retractions. The terminal relaxation times of such arms are thus much shorter than those of the arms with uniform slip-link distributions. Actually, their terminal times have been reached in the first-stage continuous simulations without going into the second stage of FFS. These $\tau_{\mathrm{d}}$ data are still counted for calculating the distribution and the mean value of the terminal relaxation times.

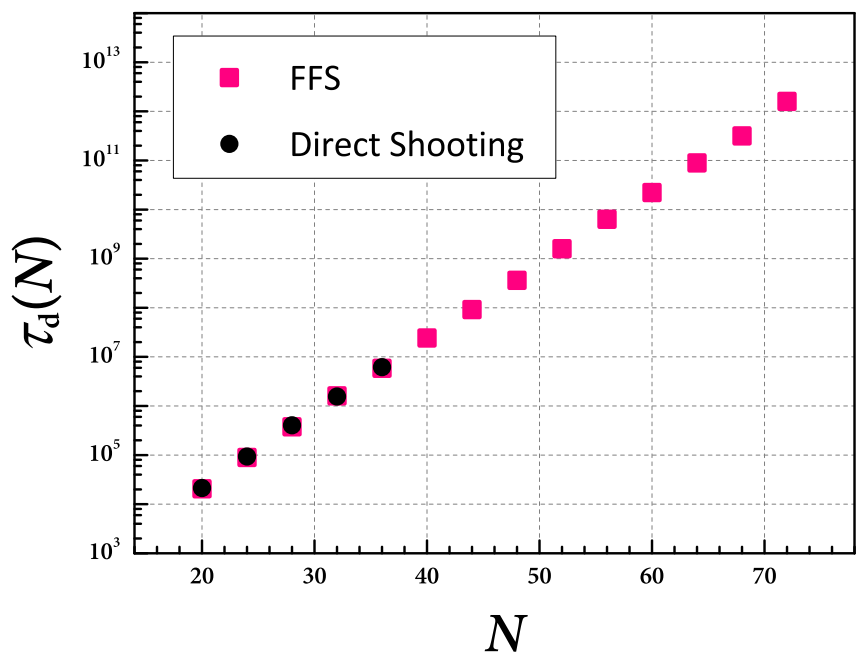

FIG. 6. Simulation results on the terminal arm retraction time $\tau_{\mathrm{d}}$ obtained from FFS and direct shooting simulations as a function of arm length $N$. 


\section{RESULTS AND DISCUSSIONS FOR SYSTEMS WITHOUT CONSTRAINT RELEASE}

\section{A. Terminal Time of Arm Retraction}

The terminal time $\tau_{\mathrm{d}}$ of the arm retraction process is the main and most straightforward output of the FFS simulations. Fig. 6 presents the FFS results on $\tau_{\mathrm{d}}$ as a function of the arm length $N$. For comparison, we have also included the $\tau_{\mathrm{d}}$ data obtained from the so-called direct shooting simulations which start from the first interface $\lambda_{0}$ and stop at the last interface $\lambda_{m}$ without intermediate steps. These runs are equivalent to the slip-spring simulations using initial configurations randomly picked from the database on interface $\lambda_{0}$ and running continuously until the innermost original slip-spring being deleted by the arm free end. For each arm length, the direct shooting simulation results are averaged over 10,000 independent samples, while in the FFS simulations $\tau_{\mathrm{d}}$ is averaged over 2,000 independent runs. Since in each FFS run, there are 40,000 samples recorded on $\lambda_{1}$, the average is actually taken over a much bigger ensemble than that of the direct shooting runs. Considering the high computational cost, the direct shooting simulations are only performed for arm lengths from $N=20$ to 36 , corresponding to about 4 to 8 entanglements per arm estimated with $N_{\mathrm{e}} \approx 4.47$ as discussed in Sec. IV B. In this range of $N$, the FFS and direct shoot simulation results in Fig. 6 show very good agreement with the relative differences less than $5 \%$. The combined FFS and SS method and the choice of the reaction coordinate are thus well justified.

Fig. 7 compares the average computational times required to complete a single direct shooting and a single FFS run on a single CPU (Intel Xeon E5-2620). The direct shooting simulation is faster at short arm lengths, but its computational time grows exponentially with $N$ and overtakes that of the FFS when $N \geq 32$. The FFS method allows us to study much longer arms. For entangled star polymers with arm length $N=72$ in the absence of $\mathrm{CR}$, the terminal relaxation time is found to be $\tau_{\mathrm{d}} \approx 2.85 \times 10^{12}$ which is about 8 orders of magnitude longer than that of stars with $N=20$ and is hardly accessible to any type of direct simulations unless running on a supercomputer for several years. 


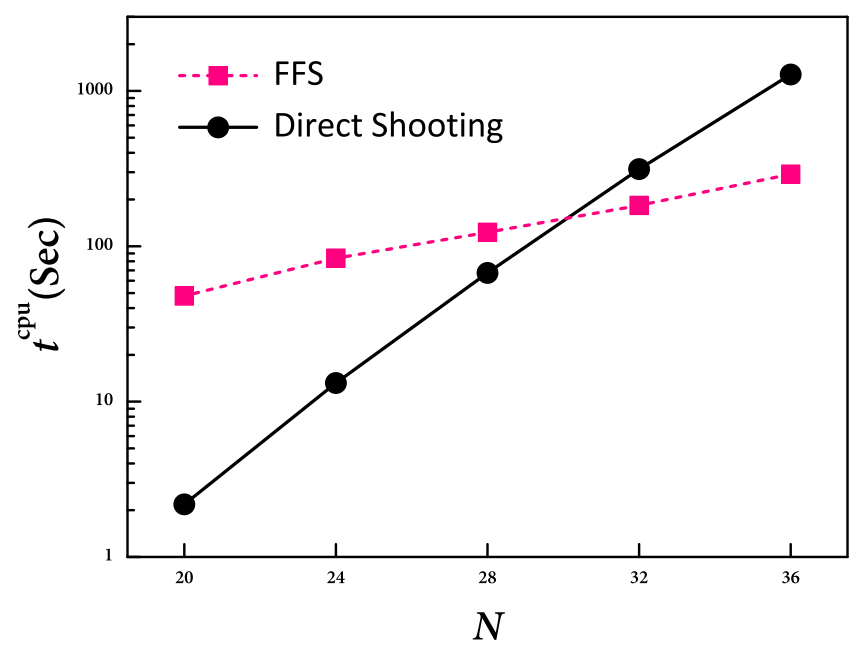

FIG. 7. Average computational times required for completing a single FFS and a single direct shooting run on a single Intel Xeon processor.

\section{B. Comparison with Theoretical Model Predictions}

The $\tau_{\mathrm{d}}$ data in Fig. 6 show a clear exponential dependence on the arm length $N$, which is expected from the Pearson-Helfand theory for star arms retracting in a fixed network. ${ }^{8}$ These results can be further compared with the predictions of more detailed theoretical models., 4,18 The Milner-McLeish theory based on the solution of 1D Kramers problem predicts the terminal arm retraction time in the absence of $\mathrm{CR}$ as ${ }^{4,5}$

$$
\tau_{\mathrm{d}}(N)=\frac{\pi^{5 / 2}}{4 \sqrt{6}} \tau_{\mathrm{R}}(N) \frac{1}{z} \exp \left(\frac{3 z^{2}}{2}\right)
$$

where $z=\sqrt{N / N_{\mathrm{e}}}$ and the arm Rouse time $\tau_{\mathrm{R}}(N)=4 \zeta_{0} N^{2} b^{2} / 3 \pi^{2} k_{\mathrm{B}} T$. The entanglement molecular weight $N_{\mathrm{e}}$ can be estimated by substituting the corresponding FFS result on $\tau_{\mathrm{d}}(N)$ into Eq. 6. As shown in Fig. 8, the obtained $N_{\mathrm{e}}$ values are roughly independent of $N$, giving $N_{\mathrm{e}} \approx 4.94$.

Recently Cao et al. pointed out that the first-passage problem of Rouse chain should be treated as a multi-dimensional Kramers problem. ${ }^{18}$ FFS simulations of 1D Rouse chains showed that the $z^{-1}$ scaling in the prefactor of $\tau_{\mathrm{d}}$ as predicted in Eq. 6 is only valid for very large chain extensions. In the intermediate chain extension regime corresponding to realistic arm retraction process, a new theory based on the Freidlin-Wentzell theory was proposed, ${ }^{54}$ 
which predicts a $z^{-3}$ scaling in the prefactor of the terminal time [Eq. 60 in Ref. ${ }^{18}$ ]

$$
\tau_{\mathrm{d}}(N)=\frac{C(N) \tau_{\mathrm{R}}(N)}{z^{3}} \exp \left(\frac{3 z^{2}}{2}\right),
$$

where $C(N)$ is a fitting parameter. For arm lengths $N \geq 20$ we can take the plateau value of $C(N)=1.2$ as found in the FFS simulations of 1D Rouse chains. ${ }^{18}$ The $N_{\mathrm{e}}$ values calculated by substituting the FFS data on $\tau_{\mathrm{d}}(N)$ into Eq. 7 are shown in Fig. 8, which increase with the increasing arm-length and approach an asymptotic value of $N_{\mathrm{e}} \approx 4.47$ that is smaller than the $N_{\mathrm{e}}$ value estimated by using Eq. 6. The two theoretical models thus predict qualitatively different dependence of $N_{\mathrm{e}}$ on $N$, at least in the systems without CR. Since the entanglement molecular weight is one of the most important model input parameters for predicting the dynamics and rheology of entangled polymers, this $N$-dependent behavior apparently needs further investigation for developing quantitative theories. The FFS results on $\tau_{\mathrm{d}}$ over a broad range of arm lengths should work as a benchmark for examining theoretical models that are typically developed for well-entangled polymers.

In Eqs. 6 and 7, the parameter $\nu$ used in the quadratic arm retraction potential is taken to be $3 / 2$ as originally proposed by Doi and Edwards for describing contour length fluctuations or arm retractions in a fixed network. ${ }^{2}$ But computer simulation and theoretical works have suggested that the value of $\nu$ actually has an arm-length dependence and even the quadratic form of the arm retraction potential may be subject to change once taking into account the enthalpic contributions. ${ }^{55,56}$ When we fit the $\tau_{\mathrm{d}}$ data in Fig. 6 to an exponential function of $\tau_{\mathrm{d}}\left(N / N_{\mathrm{e}}\right)=A \exp \left[\nu\left(N / N_{\mathrm{e}}\right)\right]$ with $N_{\mathrm{e}}=4.94$ over the whole range of arm length $N$ we studied, a value of $\nu \approx 1.69$ is found, which is somewhat larger than $3 / 2$. On the other hand, the theoretical predictions of Eq. 6 using $\nu=3 / 2$ and $N_{\mathrm{e}}=4.94$ also agree with the simulation data reasonably well. To examine the $\nu$ parameter using Eq. 7 with a fixed $N_{\text {e }}$ value could be more complicated, because this theoretical model was derived using the constant value of $\nu=3 / 2$. Considering that the simulation results in Fig. 6 are obtained in the systems without CR and the slip-spring model does not involve explicit enthalpic contributions, we keep $\nu$ as a constant in the comparison with theoretical models in the current work.

We note that the $N_{\mathrm{e}}$ values given in Fig. 8 are different from that obtained by mapping the original slip-spring model simulation results on the linear viscoelastic properties of linear polymer melts to the Likhtman-McLeish model predictions $\left(N_{\mathrm{e}} \approx 5.7\right) .{ }^{25,30}$ The difference 
could be related to the use of different theoretical models for the data fitting, the presence of constraint release effects in the polymer melts and the different ways of treating the slip-link motion along the polymer chains, namely continuously or discretely, as discussed in Sec. II A. The value of $N_{\mathrm{e}} \approx 4.94$ we found is very close to the value of $N_{e}=4.89$ estimated by Shivokhin et al. for the slip-spring model using the same set of model parameters $N_{\mathrm{e}}^{\mathrm{SS}}=4$ and $N_{\mathrm{S}}^{\mathrm{SS}}=0.5 .^{52}$

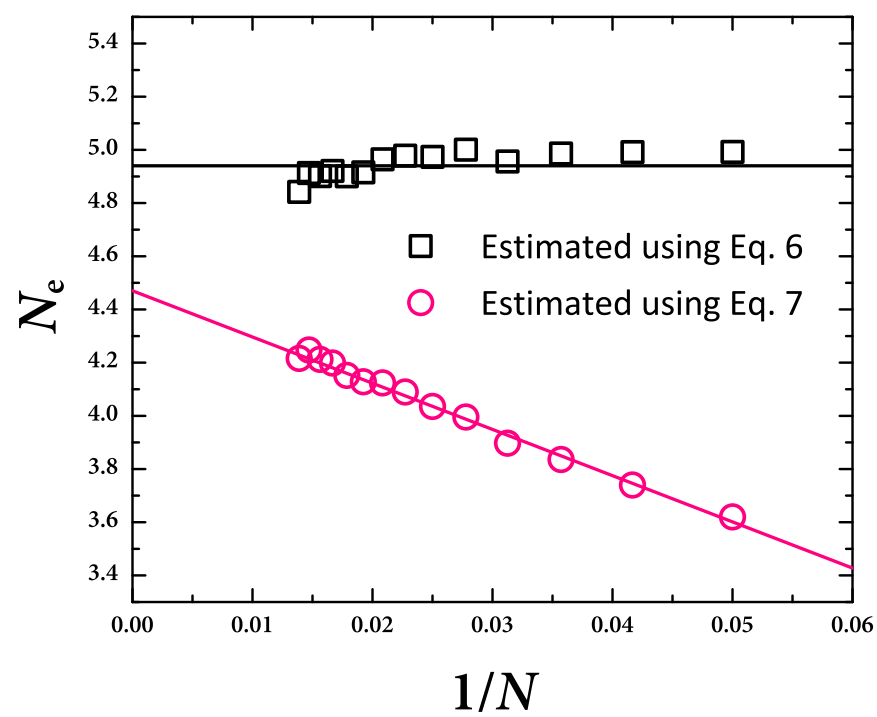

FIG. 8. Entanglement molecular weight $N_{\mathrm{e}}$ calculated by substituting the FFS simulation results on $\tau_{\mathrm{d}}$ (Fig. 6) into the theoretical predictions of Eqs. 6 (squares) and 7 (circles) for various arm lengths.

\section{Arm Relaxation Spectrum}

Apart from terminal relaxation time, the FFS method can also be applied to obtain the entire relaxation spectrum of the arm. This is done in a similar way as calculating $\tau_{\mathrm{d}}$. The only difference is to set the index of the monomer that the $i$-th original slip-link sits on, instead of that of the innermost slip-link, as the reaction coordinate. Accordingly, the first interface $\lambda_{0}$ in the FFS method is defined on the monomer where the $i$-th slip-link originally occupied. The FP time of the $i$-th slip-link is recorded as $\tau(X)$ with the fractional index $X=i /\left\langle N_{\mathrm{sl}}\right\rangle$. The simulation results on $\tau(X)$ are plotted in Fig. 9 for the arm lengths $20 \leq N \leq 44$. For the systems with $N \leq 36$, the direct shooting simulation results are 
also presented for comparison. The agreement between the FFS and direct shooting data gets improved as the arm free end retracts deeper along the primitive path, i.e., with the decrease of the slip-link index $i$ and so $X$. This is understandable because the release of the outer slip-links or entanglements is dominated by the fast Rouse-like fluctuations. The corresponding entropic barrier is relatively low such that the FFS method does not work well at large $X$. For this reason, the most reliable relaxation spectrum, especially for the long arms, should be constructed by combining the FP times of the inner slip-links as calculated by the FFS method with the FP times of the outer ones obtained from direct shooting simulations. One such example is shown in Fig. 9 for the systems with $N=44$. The complete relaxation spectrum $\tau(X)$ can be directly applied to test theoretical models of arm retraction dynamics.

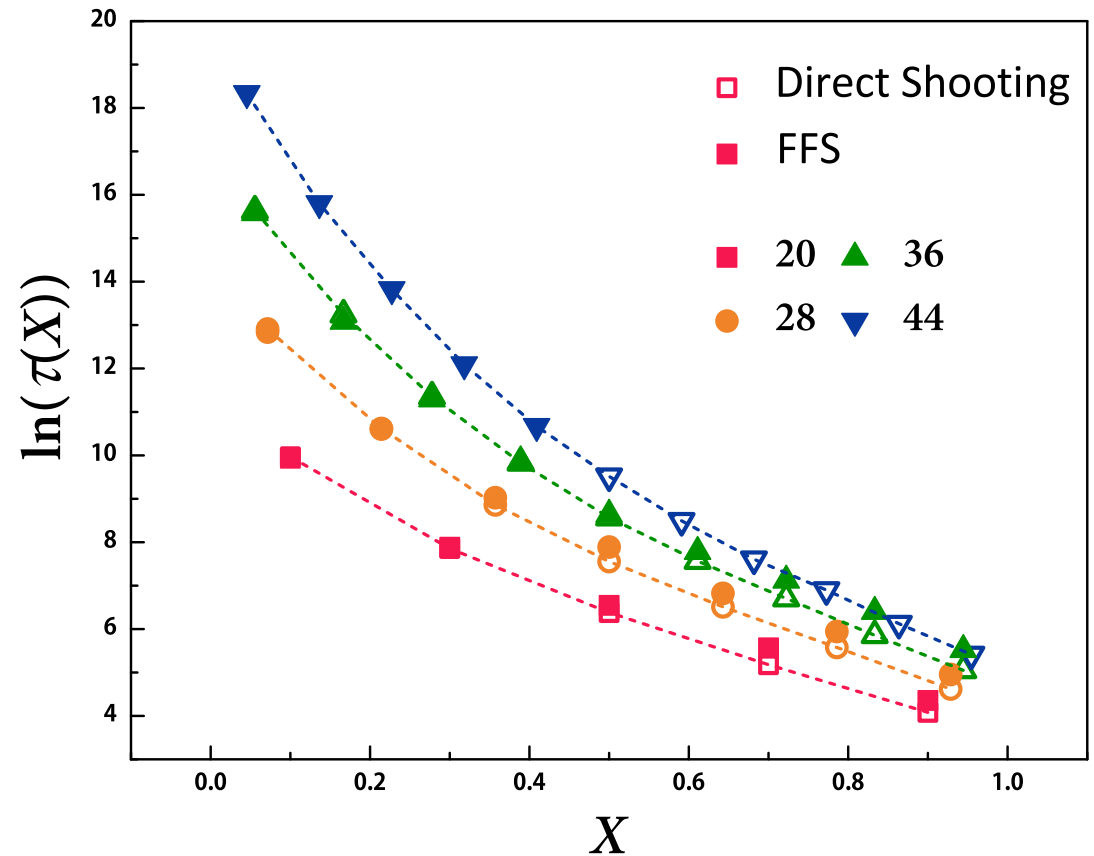

FIG. 9. Relaxation spectrum calculated using the first-passage times of all slip-links for star arms with various lengths obtained by both FFS (solid symbols) and direct shooting (open symbols) simulations. The dashed curves are for guiding the eye. The parameter $X=i /\left\langle N_{\mathrm{sl}}\right\rangle$ is the fractional index of the $i$-th slip-link along the arm, which increases from $X=1 /\left\langle N_{\mathrm{sl}}\right\rangle$ for the innermost slip-link to $1-1 /\left\langle N_{\mathrm{sl}}\right\rangle$ for the outermost one. 


\section{Constructing Relaxation Correlation Functions}

In experiments, the dynamics and rheology of entangled polymers are generally characterized by the dielectric relaxation or chain end-to-end vector correlation function, $\Phi(t)$, and the stress relaxation function, $G(t)$. The calculation of these observables usually requires the continuous trajectories of the polymers, which are however not naturally available in FFS simulations, because only instantaneous configurations at the hitting points on the interfaces are recorded. Here we introduce a numerical route to effectively link these discrete pieces of information to construct the dielectric and stress relaxation functions. The systems of entangled star polymers without $\mathrm{CR}$ are used as examples to demonstrate the application of this algorithm.

Fig. 4(b) sketches the method used to build continuous arm relaxation pathways from the piecewise FFS shooting trajectories shown in Fig. 4(a). Considering two hitting points on the terminal interface $\lambda_{m}$, marked as $A_{m}$ and $B_{m}$, there must be two continuous trajectories or pathways that one can track back from them to the first interface $\lambda_{0}$. As shown in Fig. 4(b), the pathway to state $A_{m}$ is constructed by linking the successful shooting trajectory from the hitting point $A_{m-1}$ to $A_{m}$ with that from $A_{m-2}$ to $A_{m-1}$, and so on until reaching the point $A_{1}$ on the interface $\lambda_{1}$. The linking from $A_{1}$ to a start point $A_{0}$ is obtained from the trajectory generated in the continuous simulation in the first stage of the FFS simulations. Similarly, the pathway to the hitting point $B_{m}$ can be traced back to $B_{1}$ on $\lambda_{1}$ and then to a starting point $B_{0}$. We note that these rebuilt trajectories are different from the true continuous trajectories generated in standard slip-spring model simulations, but the ensemble-averaged pathways obtained in these two cases should be very close, as reflected in the consistent $\Phi(t)$ and $G(t)$ results in Fig. 11. From computational point view, the rebuilding method requires the storage of all the successful shooting trajectories between neighboring interfaces and also a large memory for data processing. This may limit its application to large systems such as the fine-grained bead-spring models widely used in molecular dynamics simulations.

When calculating the arm relaxation correlation functions from the rebuilt trajectories, two assumptions have been made. First, when one slip-link is destroyed by the retracting arm free end, the primitive path segment in between its nearest neighboring slip-link and itself will be forgotten immediately. This assumption is valid for most of the slip-links due to 
the discrete feature of entanglements in the SS model. The only exception is with the tube segment between the branch point and the innermost slip-link where this assumption may affect the calculation of the relaxation functions, as discussed below. The second assumption is that the FP times on each interface follow a single exponential distribution. This assumption has also used in solving the 1D Kramers problem and in the Doi-Edwards tube model without $\mathrm{CR}^{2}{ }^{2}$ Since the slip-spring model is essentially a multidimensional problem, we perform an extra set of simulations to examine the validity of this assumption. A total number of 10,000 direct shooting simulations, all starting from exactly the same initial configuration, are carried out to mimic a FFS run. The FP times for the innermost slip-link to reach different monomers, or different interfaces in the FFS definition, are recorded. Fig. 10 presents the probability distributions, $P_{i}(t)$, of the FP times on three different interfaces for the arms of length $N=20$. It can be seen that $P_{i}(t)$ on interfaces with higher indexes can be well described by the exponential function

$$
P_{i}(t)=\frac{1}{\tau_{i}} \exp \left(-\frac{t}{\tau_{i}}\right)
$$

where $\tau_{i}$ is the mean FP time on the interface $\lambda_{i}$. The second assumption becomes valid as the arm free end retracts deeply along the primitive path.

Following Eq. 8 the probability that the innermost slip-link has never crossed the interface $\lambda_{i}$ after time $t$ is

$$
P_{\lambda_{0}}^{\lambda_{i}}(t)=\exp \left(-\frac{t}{\tau_{i}}\right), \quad i=1,2, \ldots, m
$$

and the probability that it has crossed $\lambda_{i}$ at least once is

$$
P_{\lambda_{i}}^{\infty}(t)=1-\exp \left(-\frac{t}{\tau_{i}}\right), \quad i=1,2, \ldots, m .
$$

Therefore the probability that the trajectory starting from $\lambda_{0}$ has crossed interface $\lambda_{i}$ but never crossed interface $\lambda_{i+1}$ is

$$
P_{\lambda_{i}}^{\lambda_{i+1}}(t)=P_{\lambda_{i}}^{\infty}(t)-P_{\lambda_{i+1}}^{\infty}(t)=-\exp \left(-\frac{t}{\tau_{i}}\right)+\exp \left(-\frac{t}{\tau_{i+1}}\right), \quad i=1,2, \ldots, m-1 .
$$

Using Eqs. 9, 10 and 11, the time correlation function of a dynamic observable, $V$, whose instantaneous values are calculated on different interfaces can be evaluated by

$$
\langle V(t) V(0)\rangle=\left\langle P_{\lambda_{0}}^{\lambda_{1}}(t) W_{0}+\sum_{i=1}^{m-1} P_{\lambda_{i}}^{\lambda_{i+1}}(t) W_{i}+P_{\lambda_{m}}^{\infty}(t) W_{m}\right\rangle
$$




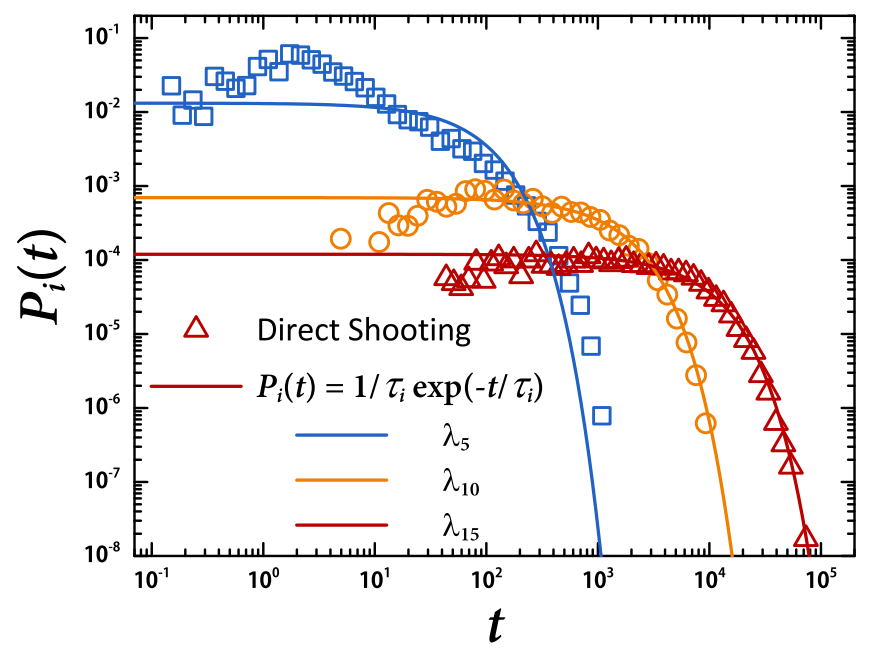

FIG. 10. Probability distributions of the first-passage times for the innermost slip-link to reach different monomers or different interfaces in the FFS definition $\lambda_{i}$ along the arm as calculated by direct shooting slip-spring simulations of star arms of length $N=20$. All of the 10,000 simulations start from the same initial configuration where the innermost slip-link sits on monomer 1 next to the branch point. The solid lines represent a single exponential fit to the simulation data in each case.

where $W_{i}$ is defined as

$$
W_{i}=\frac{1}{h_{i}} \sum_{k=1}^{h_{i}} V_{i}^{k} V_{0}^{k}, \quad i=0,1, \ldots, m .
$$

Here $h_{0}$ is the number of starting points on the first interface $\lambda_{0}$ and $V_{0}^{k}$ is the observable value at the $k$-th starting point. Similarly $h_{i}(i=1, \ldots, m)$ is the number of hitting points on the interface $\lambda_{i}$ out of the $M_{i-1}$ shootings from $\lambda_{i-1}$ and $V_{i}^{k}$ is the observable value at the $k$-th hitting point on $\lambda_{i}$, respectively. For the system sketched in Fig. 4(b), there are only 2 hitting points on the final interface $\lambda_{m}$ such that $h_{m}=2$ in Eq. 13 .

Substituting Eqs. 10 and 11 into Eq. 12, we get

$$
\langle V(t) V(0)\rangle=\left\langle\sum_{i=0}^{m-1} \Delta W_{i, i+1} \exp \left(-\frac{t}{\tau_{i+1}}\right)+W_{m}\right\rangle,
$$

where $\Delta W_{i, i+1}=W_{i}-W_{i+1}$. The correlation function in Eq. 14 is expressed as a weighted summation of a set of exponential functions, which is consistent with the tube model predictions for the end-to-end vector and stress relaxation functions of entangled polymers in the absence of constraint release. ${ }^{2}$ The only difference lies in the last term $W_{m}$ on the right 
hand side of Eq. 14 which, if being nonzero, may result in an unphysical plateau after the terminal relaxation time $\tau_{\mathrm{d}}$.

The problem associated with $W_{m}$ does not exist in the tube model where the tube is assumed to be continuous. ${ }^{2}$ The arm free end can thus retract continuously along the primitive path all the way to the branch point and so release all the memories in the original tube. As a result, $W_{m}$ equals to zero for all dynamic observables. However, in the slip-spring model the entanglements are represented discretely by the slip-links. The terminal time $\tau_{\mathrm{d}}$ is taken to be the time when the arm free end passes the innermost slip-link. In standard slip-spring model simulations, the memories, such as stress and arm end-to-end vector orientation, stored in the original tube segment between the innermost slip-link and the branch point can still be released by the continuous relaxation of the arm beyond $\tau_{\mathrm{d}}$. But in the FFS simulations, the runs are terminated right after $\tau_{\mathrm{d}}$ when the trajectories reach the last interface $\lambda_{m}$. Although this termination does not affect the determination of the terminal time as shown above, it artificially traps the unreleased memories in the last tube segment in the configurations saved on $\lambda_{m}$, leading to a nonzero ensemble average value of $W_{m}$. As an attempt to recover the full relaxation function, we propose a simple approximation to incorporate the arm relaxation dynamics beyond the terminal time $\tau_{\mathrm{d}}\left(=\tau_{m}\right)$, which is to multiply the $W_{m}$ term in Eq. 14 with an exponential time decay function, giving

$$
\langle V(t) V(0)\rangle=\left\langle\sum_{i=0}^{m-1} \Delta W_{i, i+1} \exp \left(-\frac{t}{\tau_{i+1}}\right)+W_{m} \exp \left(-\frac{t}{\tau_{m}}\right)\right\rangle .
$$

The dielectric and stress relaxation functions calculated using Eq. 15 from the rebuilt trajectories are plotted in Fig. 11 for arm lengths up to $N=72$. For comparison, the $\Phi(t)$ and $G(t)$ data obtained from standard slip-spring model simulations are also included for the systems with $N \leq 36$. In these calculations, the dielectric or arm end-to-end vector relaxation function is defined as $\Phi(t)=\left\langle\boldsymbol{R}_{\mathrm{e}}(t) \cdot \boldsymbol{R}_{\mathrm{e}}(0)\right\rangle /\left\langle\boldsymbol{R}_{\mathrm{e}}^{2}(0)\right\rangle$ where $\boldsymbol{R}_{\mathrm{e}}$ is the arm endto-end vector and the mean square end-to-end distance $\left\langle\boldsymbol{R}_{\mathrm{e}}^{2}(0)\right\rangle=N b^{2}$. The $G(t)$ results are the single-arm stress autocorrelation functions without considering the cross-correlation contributions from the virtual springs. ${ }^{57,58}$ This choice does not affect any discussions or conclusions in the current work, especially when there is no constraint release effect. The $\Phi(t)$ and $G(t)$ results obtained by using the rebuilding method and from the standard SS model simulations show reasonably good agreement in the terminal regime, indicating the capability of Eq. 15 in constructing the arm relaxation functions using discrete FFS shooting 
trajectories. The noticeable discrepancy between the two sets of data in each case at short time scales could be attributed to the fact that the exponential distribution assumption of the FP times does not apply to the first few interfaces, as shown in Fig. 10. On the other hand, we have also applied Eq. 14 directly to construct the relaxation functions of the systems with $N=36$. The obtained $\Phi(t)$ and $G(t)$ curves (dashed lines) initially coincide with those calculated using Eq. 15, but start to decay slower when some of the sample trajectories have reached their terminal times and the constant $W_{m}$ contributions are counted in. The unphysical plateaus are reached after the mean terminal time $\tau_{\mathrm{d}}$ for the reasons discussed above. Therefore at least for the combined FFS and SS method we used, the algorithm for constructing the time correlation functions needs to take into account the arm relaxation behavior beyond $\tau_{\mathrm{d}}$.

\section{EXTENSION OF THE COMBINED FFS AND SS METHOD TO SYSTEMS WITH CONSTRAINT RELEASE}

The combined FFS and SS method can be extended to entangled polymer systems with CR by adjusting the definition of the reaction coordinate. In the standard slip-spring model ${ }^{25,51}$ constraint release is included by coupling the slip-links sitting on different polymer chains or arms into pairs to represent the binary entanglements. When one slip-link is deleted from the free end of an arm, its coupled partner is also deleted regardless of its location, which results in a CR event. This means that for FFS simulations the originally innermost slip-link alone could not be used to define a reaction coordinate for exploring the entire arm relaxation spectrum, because this slip-link may be destructed by a CR event before reaching the arm free end. To resolve this problem, we refer to a recent slip-spring simulation work on entangled symmetric star polymers with CR. ${ }^{51}$ There it was shown that the relaxation of the original tube segments, and correspondingly the relaxation of the arm end-to-end vector, is dominated by the first-passage times of the so-called tube-representative (TR) slip-links, which are the original slip-links finally released from the arm free end. The other original slip-links which are destructed from the middle of the arm by CR events only contribute to stress relaxation. For determining the terminal relaxation time of the arm end-to-end vector, we only need to find the moment when the last tube segment held in between the branch point and the innermost TR slip-link is released by the arm free end. 

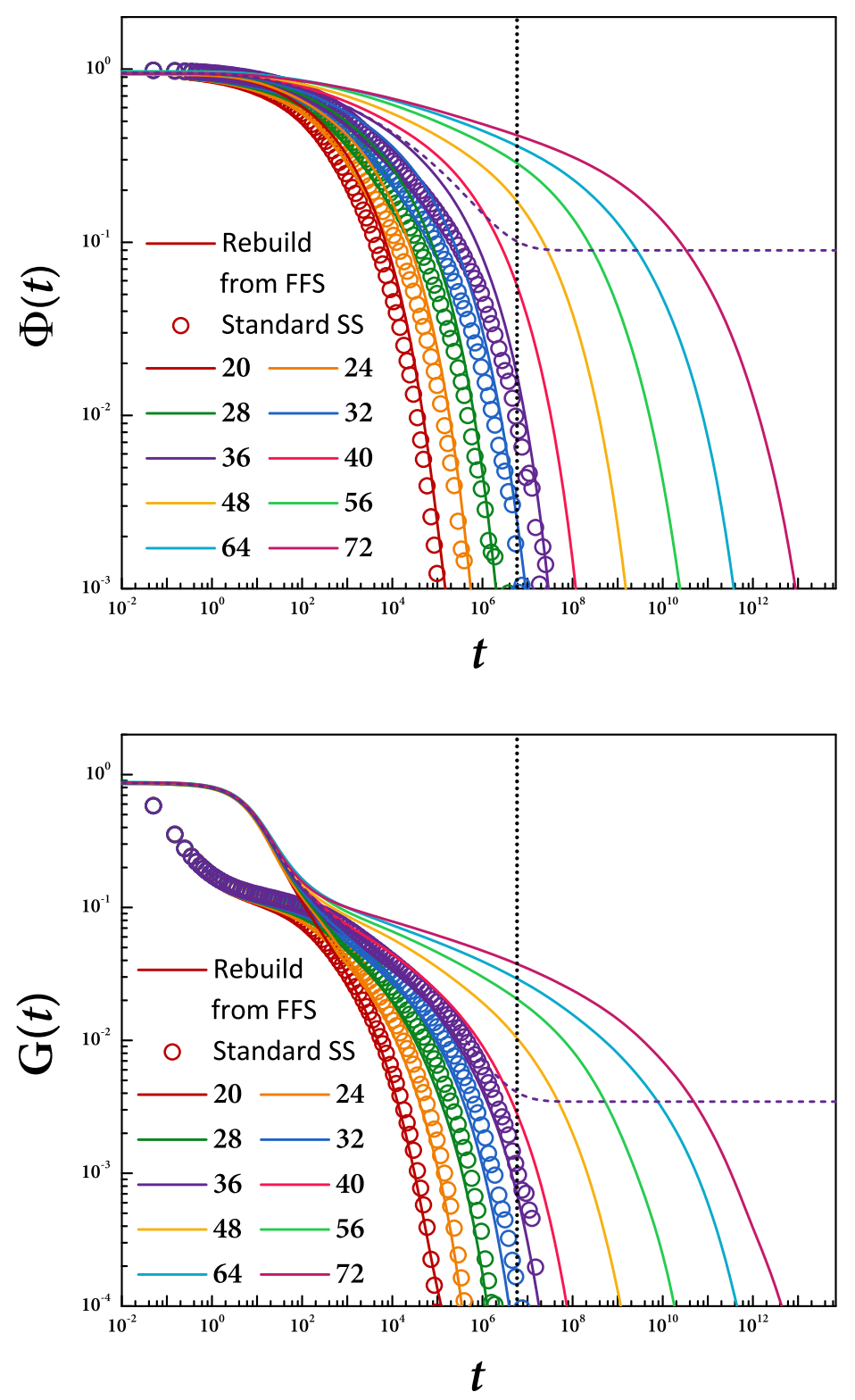

FIG. 11. (a) Arm end-to-end vector correlation function $\Phi(t)$ and (b) stress relaxation function $G(t)$ obtained from standard slip-spring simulations (symbols) and calculated using Eq. 15 in the revised manuscript from the rebuilt trajectories (solid lines), respectively. The dashed lines represent the results on the systems with arm length $N=36$ calculated by using Eq. 14 directly with the $W_{m}$ term included. The vertical dotted lines mark the terminal relaxation time $\tau_{\mathrm{d}}$ of arms with $N=36$ as determined in the FFS simulations. 
Since it is not known in advance whether an original slip-link will be deleted by the arm end or by $\mathrm{CR}$, we can define the reaction coordinate as the index of the monomer that the innermost surviving original slip-link sits on. In other words, if at time $t$ the innermost original slip-link was deleted by $\mathrm{CR}$, the reaction coordinate will be immediately shifted from the monomer it sat on to the monomer occupied by the nearest original slip-link, because the latter becomes the innermost surviving original slip-link.

Different from the systems without CR where each star arm is treated independently, the FFS simulations of the systems with CR require the use of an ensemble of star polymers where the slip-links sitting on different arms are coupled with each other. In the current work, the simulated system consists of 20 three-arm star polymers with a total number of $N_{\text {arm }}=60$ arms. The branch points of the stars are allowed to move in space. Only one randomly chosen arm out of the whole ensemble is used for the FFS study. The setup of the interfaces on this target arm is similar to that used in the non-CR case (Fig. 5). The first interface $\lambda_{0}$ is set on the monomer that the initially innermost slip-link along this arm sits on, and the subsequent interfaces are placed on outer monomers with the intervals of $l_{1}=2$ and $l_{2}=1$. The reaction coordinate is defined as the index of the monomer where the innermost surviving original slip-link sits on. Both the first-stage continuous and the second-stage shooting simulations are run as the standard slip-spring model simulations which involve all star polymers in the ensemble to allow for constraint release. It means that the configurations of all these polymers need to be stored in the database on each interface. If there is no reaction coordinate jumping due to $\mathrm{CR}$, the shooting simulations are carried out in the same way as in the non-CR case from interface $\lambda_{i}$ to $\lambda_{i+1}$ for $i=1, \ldots, m-1$. But if during a shooting simulation started from interface $\lambda_{i}$, a CR event causes the jump of the reaction coordinate from the destructed innermost original slip-link to the nearest surviving original slip-link, the trajectory may immediately cross one or more interfaces. In this case we allow the simulation to continue until reaching the next interface, say $\lambda_{i+j}$ with $j \geq 2$, and then save the configuration of the system in the database of interface $\lambda_{i+1}$ (instead of $\lambda_{i+j}$ ). When a shooting simulation from $\lambda_{i+1}$ selects this configuration as its starting point, the trajectory will instantaneously reach the next interface $\lambda_{i+2}$, because the reaction coordinate has actually reached or crossed this interface. As a result of the successful shooting, the same configuration will be saved in the database of $\lambda_{i+2}$. Following similar shooting and saving processes, this configuration will be stored in the databases of all relevant interfaces 
from $\lambda_{i+1}$ to $\lambda_{i+j}$ for further sampling. This approach ensures that the events that this jumping trajectory has also successfully crossed the interfaces $\lambda_{i+1}, \ldots, \lambda_{i+j-1}$ are correctly counted for calculating the transition probabilities between different interfaces. The FFS run is terminated until the last surviving original slip-link is destructed by the arm free end and so the terminal relaxation time $\tau_{\mathrm{d}}$ is reached. In each FFS run there are 20,000 samples recorded on each interface $\lambda_{i}(i=1, \ldots, m-1)$, and the final results on $\tau_{\mathrm{d}}$ are averaged over 1000 independent FFS runs. In the current method for the CR case, although the simulations and data storage involve an ensemble of $N_{\text {arm }}$ arms, only the relaxation spectrum of the target arm can be collected in each FFS sample run. The computational cost and memory storage requirement are thus still high for simulating systems with very long arms. Further improvement in the efficiency of the algorithm is apparently needed. Another possible direction is to use the single-chain slip-spring or slip-link models with self-consistent treatment of constraint release. ${ }^{59}$

The ensemble-averaged terminal relaxation times, $\tau_{\mathrm{d}}$, obtained in the FFS simulations with the modified definition of the reaction coordinate are presented in Fig. 12, together with the terminal relaxation times of the arm end-to-end vector relaxation functions as obtained from standard slip-spring model simulations and the mean FP times of the innermost surviving original slip-links as obtained from the direct shooting simulations. The three sets of data show very good agreement within error bars, which effectively validates the proposed FFS method. The combined FFS and SS method can thus provide quantitative predictions on the terminal relaxation times of entangled star polymers either with or without CR over a broad range of arm lengths that are surely needed for the development of quantitative theories for entangled branched polymers. The construction of the relaxation correlation functions, $\Phi(t)$ and $G(t)$, in the CR cases is rather complicated and will be left for later studies.

\section{CONCLUSIONS}

We present an application of the forward flux sampling method in combination with the slip-spring model on studying the arm retraction dynamics of entangled star polymers. The single-chain slip-spring model originally developed for describing entangled linear polymers has been extended to model symmetric star polymers. As a proof of concept, we start 


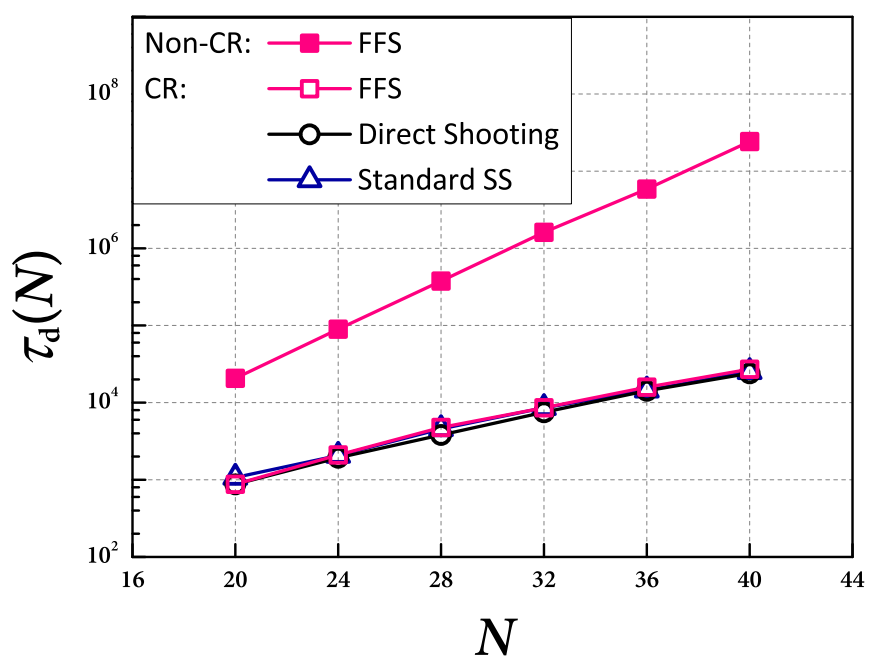

FIG. 12. Simulation results on the terminal arm relaxation times $\tau_{\mathrm{d}}$ obtained from the FFS (open squares) and direct shooting (open circles) simulations, together with the terminal times of the arm end-to-end vector correction functions calculated from standard slip-spring simulations (open triangles), in the systems with constraint release. For reference, the FFS results on $\tau_{\mathrm{d}}$ for the systems without CR (solid squares, same as in Fig. 6) are also plotted.

with the systems without constraint release where the entanglements or slip-links can only be created on or deleted from the arm free ends, making the FFS method conveniently applicable. Two possible reaction coordinates for the FFS simulations have been tested. The choice of the index of the monomer that the originally innermost slip-link sits on is found to provide FFS simulation results on terminal relaxation times $\tau_{\mathrm{d}}$ in good agreement with those obtained in direct shooting simulations for mildly entangled stars with arm lengths up to 8 entanglements. The FFS simulations are then performed to study the terminal relaxation of much longer arms (up to 16 entanglements) that are hardly accessible by any direct simulations, especially considering the exponential growth of $\tau_{\mathrm{d}}$ with the arm length in the absence of CR. The FFS results on $\tau_{\mathrm{d}}$ over such a broad range of arm lengths allow direct comparison with the predictions of theoretical models which are typically developed for well-entangled polymers. The entanglement molecular weight $N_{\mathrm{e}}$ extracted from such comparison is found to have an arm-length dependence.

In addition to the terminal arm relaxation time, the first-passage times of all other original slip-links on a given arm can also be conveniently calculated by defining the reaction coordinate as the index of the monomer that the interested slip-link sits on, which in turn 
provides the entire relaxation spectrum of the arm. For mildly entangled arms the FFS results on the FP times show good agreement with direct shooting simulation data for the deep entanglements or inner slip-links, but some discrepancy exists for the shallow ones, because the FFS method does not work well at low entropic barriers. The reliable relaxation spectrum of long star arms thus should be constructed by combining the FP times of the inner slip-links as calculated by the FFS method with the FP times of the outer ones obtained from direct simulations. Furthermore, we have proposed a numerical route to construct the arm relaxation correlation functions from the FFS simulation data saved on discrete interfaces. This method is essentially a summation of weighted exponential relaxation functions with characteristic times determined by the mean FP times of different slip-links along the arm. The so-constructed arm end-to-end vector correlation functions, $\Phi(t)$, and stress relaxation functions, $G(t)$, show reasonably good agreement with those obtained in standard slip-spring simulations in the terminal regime, while the noticeable discrepancy at short time scales can be attributed to the use of a too strong assumption that the first-passage times at the first few FFS interfaces follow the exponential distribution.

We have also attempted to extend the FFS method to systems with constraint release, namely to entangled star polymer melts. The key change from the non-CR case is to define the reaction coordinate using the innermost surviving original slip-link. Again good agreement is found between the FFS simulation results on the terminal arm relaxation time with those obtained in standard slip-spring model simulations. Therefore the combined FFS and slip-spring simulation method provides an efficient tool for studying the dynamics of highly entangled branched polymers which are generally inaccessible to direct simulation methods but highly desired for the development of quantitative theories on entangled branched polymers.

\section{Author Information}

Corresponding Author: * Email: zuowei.wang@reading.ac.uk

\section{ACKNOWLEDGMENTS}

We thank Daniel Read and Patrick Ilg for helpful discussions. This work was supported by the Engineering and Physical Sciences Research Council (EPSRC), Grant EP/K017683/1. 


\section{REFERENCES}

${ }^{1}$ P. G. de Gennes, J. Chem. Phys. 55, 572 (1971).

${ }^{2}$ M. Doi and S. F. Edwards, The Theory of Polymer Dynamics (Oxford University Press, 1988).

${ }^{3}$ A. E. Likhtman and T. C. B. McLeish, Macromolecules 35, 6332 (2002).

${ }^{4}$ S. Milner and T. McLeish, Macromolecules 30, 2159 (1997).

${ }^{5}$ S. Milner and T. McLeish, Phys. Rev. Lett. 81, 725 (1998).

${ }^{6}$ S. T. Milner and J. D. Newhall, Phys. Rev. Lett. 105, 208302 (2010).

${ }^{7}$ M. Doi and N. Y. Kuzuu, J. Polym. Sci., Polym. Lett. Ed. 18, 775 (1980).

${ }^{8}$ D. S. Pearson and E. Helfand, Macromolecules 17, 888 (1984).

${ }^{9}$ R. Ball and T. McLeish, Macromolecules 22, 1911 (1989).

${ }^{10}$ G. Marrucci, J. Polym. Sci., Polym. Phys. Ed. 23, 159 (1985).

${ }^{11}$ A. L. Frischknecht, S. T. Milner, A. Pryke, R. N. Young, R. Hawkins, and T. C. B. McLeish, Macromolecules 35, 4801 (2002).

${ }^{12}$ R. G. Larson, Macromolecules 34, 4556 (2001).

${ }^{13}$ S. J. Park, S. Shanbhag, and R. G. Larson, Rehol. Acta 44, 319 (2005).

${ }^{14}$ C. Das, N. J. Inkson, D. J. Read, M. A. Kelmanson, and T. C. B. McLeish, J. Rheol. 50, 207 (2006).

${ }^{15}$ E. van Ruymbeke, C. Bailly, R. Keunings, and D. Vlassopoulos, Macromolecules 39,6248 (2006).

${ }^{16}$ Z. Wang, X. Chen, and R. G. Larson, J. Rheol. 54, 223 (2010).

${ }^{17}$ P. S. Desai, B.-G. Kang, M. Katzarova, R. Hall, Q. Huang, S. Lee, M. Shivokhin, T. Chang, D. C. Venerus, J. Mays, J. D. Schieber, and R. G. Larson, Macromolecules 49, 4964 (2016). ${ }^{18}$ J. Cao, J. Zhu, Z. Wang, and A. E. Likhtman, J. Chem. Phys. 143, 204105 (2015).

${ }^{19}$ C. C. Hua and J. D. Schieber, J. Chem. Phys. 109, 10018 (1998).

${ }^{20}$ Y. Masubuchi, J.-I. Takimoto, K. Koyama, G. Ianniruberto, G. Marrucci, and F. Greco, J. Chem. Phys. 115, 4387 (2001).

${ }^{21}$ J. D. Schieber, J. Neergaard, and S. Gupta, J. Rheol. 47, 213 (2003).

${ }^{22}$ Y. Masubuchi, G. Ianniruberto, F. Greco, and G. Marrucci, J. Chem. Phys. 119, 6925 (2003).

${ }^{23}$ Y. Masubuchi, G. Ianniruberto, F. Greco, and G. Marrucci, Modell. Simul. Mater. Sci. 
Eng. 12, S91 (2004).

${ }^{24}$ T. Yaoita, T. Isaki, Y. Masubuchi, H. Watanabe, G. Ianniruberto, F. Greco, and G. Marrucci, J. Chem. Phys. 121, 12650 (2004).

${ }^{25}$ A. E. Likhtman, Macromolecules 38, 6128 (2005).

${ }^{26}$ M. Shivokhin, E. Van Ruymbeke, C. Bailly, D. Kouloumasis, N. Hadjichristidis, and A. E. Likhtman, Macromolecules 47, 2451 (2014).

${ }^{27}$ E. Pilyugina, M. Andreev, and J. D. Schieber, Macromolecules 45, 5728 (2012).

${ }^{28}$ V. C. Chappa, D. C. Morse, A. Zippelius, and M. Müller, Phys. Rev. Lett. 109, 148302 (2012).

${ }^{29}$ S. K. Sukumaran and A. E. Likhtman, Macromolecules 42, 4300 (2009).

${ }^{30}$ Z. Wang, A. E. Likhtman, and R. G. Larson, Macromolecules 45, 3557 (2012).

${ }^{31}$ M. Wang, A. E. Likhtman, and B. D. Olsen, ACS Macro Lett. 4, 242 (2015).

${ }^{32}$ A. E. Likhtman, in Polymer Science: A Comprehensive Reference (Elsevier B.V., 2012).

${ }^{33}$ A. V. Brukhno, J. Anwar, R. Davidchack, and R. Handel, J. Phys. Condens. Matter 20, 494243 (2008).

${ }^{34}$ D. Quigley and P. M. Rodger, J. Chem. Phys. 128, 154518 (2008).

${ }^{35}$ R. J. Allen, P. B. Warren, and P. R. Ten Wolde, Phys. Rev. Lett. 94, 018104 (2005).

${ }^{36}$ A. Borgia, P. M. Williams, and J. Clarke, Annu. Rev. Biochem. 77, 101 (2008).

${ }^{37}$ T. Li, D. Donadio, G. Russo, and G. Galli, Phys. Chem. Chem. Phys. 13, 19807 (2011).

${ }^{38}$ G. M. Torrie and J. P. Valleau, J. Comput. Phys. 23, 187 (1977).

${ }^{39}$ C. Dellago, P. G. Bolhuis, F. S. Csajka, and D. Chandler, J. Chem. Phys. 108, 1964 (1998).

${ }^{40}$ R. J. Allen, C. Valeriani, and P. R. ten Wolde, J. Phys. Condens. Matter 21, 463102 (2009).

${ }^{41}$ K. Kratzer, A. Arnold, and R. J. Allen, J. Chem. Phys. 138, 164112 (2013).

${ }^{42}$ E. E. Borrero and F. A. Escobedo, J. Chem. Phys. 125, 164904 (2006).

${ }^{43}$ J. T. Berryman and T. Schilling, J. Chem. Phys. 133, 244101 (2010).

${ }^{44}$ N. B. Becker, R. J. Allen, and P. R. ten Wolde, J. Chem. Phys. 136, 05B607 (2012).

${ }^{45}$ B. W. Zhang, D. Jasnow, and D. M. Zuckerman, Proc. Natl. Acad. Sci. 104, 18043 (2007).

${ }^{46}$ B. W. Zhang, D. Jasnow, and D. M. Zuckerman, J. Chem. Phys. 132, 054107 (2010).

${ }^{47}$ G. A. Huber and S. Kim, Biophys. J. 70, 97 (1996).

${ }^{48}$ P. E. Rouse Jr, J. Chem. Phys. 21, 1272 (1953). 
${ }^{49}$ A. E. Likhtman and M. Ponmurugan, Macromolecules 47, 1470 (2014).

${ }^{50}$ J. Qin and S. T. Milner, Macromolecules 47, 6077 (2014).

${ }^{51}$ J. Cao and Z. Wang, Macromolecules 49, 5677 (2016).

730

${ }^{52}$ M. Shivokhin, D. Read, D. Kouloumasis, R. Kocen, F. Zhuge, C. Bailly, N. Hadjichristidis,

731 and A. Likhtman, Macromolecules, In Press (2017).

732

${ }^{53}$ J. D. Schieber, J. Chem. Phys. 118, 5162 (2003).

733

${ }^{54}$ M. Freidlin, J. Szucs, and A. Wentzell, Random Perturbations of Dynamical Systems (Springer New York, 2012).

735

${ }^{55}$ T. C. B. McLeish, Advances in Phys. 51, 1379 (2002).

${ }^{56}$ R. N. Khaliullin and J. D. Schieber, Phys. Rev. Lett. 100, 188302 (2008).

${ }^{57}$ J. Ramirez, S. K. Sukumaran, and A. E. Likhtman, J. Chem. Phys. 126, 244904 (2007).

${ }^{58}$ T. Uneyama and Y. Masubuchi, J. Chem. Phys. 137, 154902 (2012).

739

${ }^{59}$ R. N. Khaliullin and J. D. Schieber, Macromolecules 42, 7504 (2009). 